\title{
Accelerated Production Without Powder Carry-Over
}

\author{
The installation of an infrared booster in the entrance area of an existing infrared oven allowed Smart \\ Architectural Aluminium to increase the speed of its powder coating production line by 20 percent. \\ The booster helps to prevent powder carry-over and to improve the quality of the premium \\ aluminium profiles even further.
}

Smart Architectural Aluminium is the largest manufacturer of aluminium profiles for windows, doors, facades and conservatories in the United Kingdom. At its plant in Yatton near Bristol, it coats aluminium profiles on two vertical lines and one horizontal line. Here a powder coating is applied to the profiles, which are then transported through gas catalytic infrared ovens. These cause the powder to gel before it is finally cured in a convection oven.

The gas catalytic infrared panels for the horizontal line were installed about ten years ago. During this time, loose powder had made its way into the oven from the entrance area and contaminated the panels, which reduced their efficiency. This in turn caused quality problems in the production process.

Heraeus Noblelight suggested that instead of simply replacing the gas catalytic panels, Smart Architectural Aluminium could install an additional infrared booster ahead of the oven. The booster would use heat to make the powder start to gel more quickly and would prevent any carry-over of powder onto the gas catalytic panels in the oven. The powder would gel fully in the oven and the parts would then be transported to the convection oven for final curing.

The team at Smart Architectural Aluminium were impressed by this plan, particularly as the infrared booster could easily be fitted to the existing steel struc-

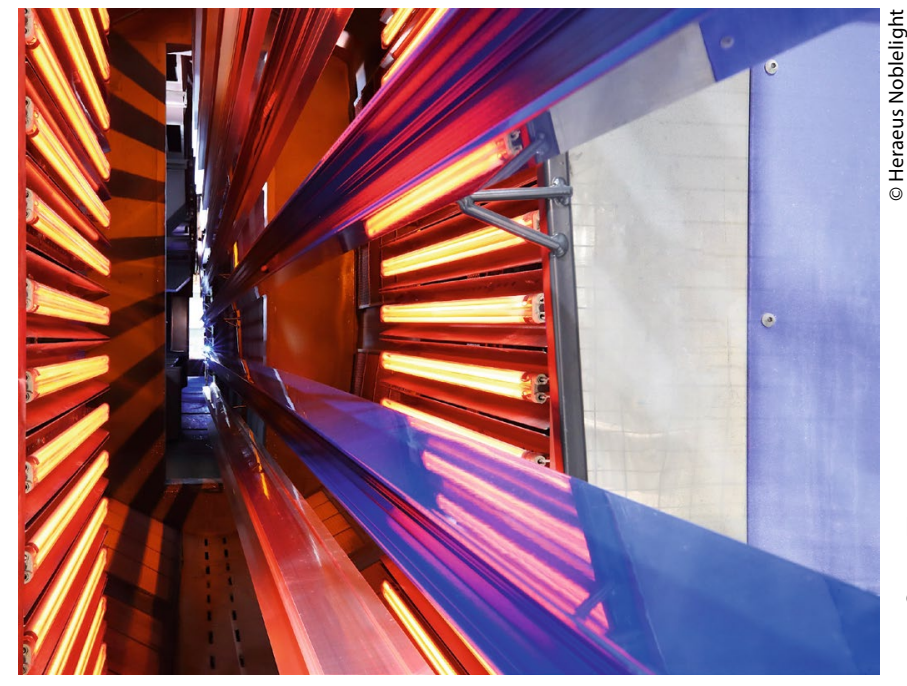

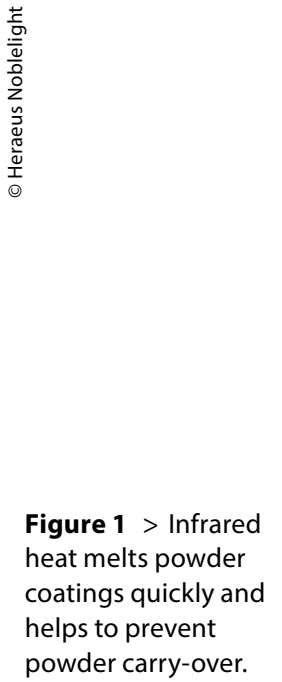

ture in the entrance area of the gas catalytic oven. Since the installation of the medium-wave infrared emitters, which are mounted in an aluminium frame with stainless steel reflectors, the system has been operating successfully.

\section{Efficient cross-linking using less energy}

Powder coatings generally absorb infrared radiation very well. Therefore, the powder heats up rapidly and forms a gel much more quickly than in a convection oven. Because there is no air movement, no dust lands on the surfaces and the powder is not disturbed or carried over. Fast melting improves the quality of the coating and increases the throughput speed.

As Michael Coles, production manager at Smart, explains: "The infrared booster was easy to fit in the existing space in the entrance area and has given us a simple but elegant solution to a potential contamination problem, extended the service life of the panels and improved the quality of the product." //

\section{Contact}

Heraeus Noblelight GmbH

Kleinostheim (Germany)

hng-infrared@heraeus.com

www.heraeus-noblelight.com/infrared 